\title{
СТРУКТУРА ТРЕВОЖНЫХ ПЕРЕЖИВАНИЙ, АССОЦИИРОВАННЫХ С РАСПРОСТРАНЕНИЕМ COVID-19: ДАННЫЕ ОНЛАЙН-ОПРОСА
}

\author{
М. Ю. Сорокин ${ }^{1 凶}$, Е. Д. Касьянов ${ }^{1}$, Г. В. Рукавишников ${ }^{1}$, О. В. Макаревич ${ }^{1}$, Н. Г. Незнанов ${ }^{1,2}$, Н. Б. Лутова, Г. Э. Мазо
}

${ }^{1}$ Национальный медицинский исследовательский центр психиатрии и неврологии имени В. М. Бехтерева, Санкт-Петербург, Россия

2 Первый Санкт-Петербургский государственный медицинский университет имени академика И. П. Павлова, Санкт-Петербург, Россия

\begin{abstract}
Пандемия COVID-19 не только создала серьезные угрозы для физического здоровья населения, но и вызвала широкий спектр психологических проблем. Целью работы было выявить структуру тревожных переживаний населения в период эпидемии и определить наиболее уязвимые социальные группы (в том числе среди лиц с аффективными расстройствами), больше всего нуждающиеся в психологической и/или психиатрической помощи. Было проведено онлайн-анкетирование 1957 русскоговорящих респондентов старше 18 лет в период с 30 марта по 5 апреля 2020 г. Уровень тревожного дистресса верифицировали по шкале психологического стресса (PSM-25), стигматизацию лиц с респираторными симптомами по модифицированному опроснику обесценивания/дискриминации (PDD; Cronbach's $\alpha=0,707)$. У 99,8\% респондентов обнаружено сочетание нескольких типов тревожных переживаний о COVID-19, показатель стресса был повышен до уровня средней интенсивности (104,9 \pm 34,4 балла), а показатель стигматизации превосходил медианное значение по выборке (19,5 \pm 3,4; Ме = 17). До 35\% респондентов имели опасения, ассоциированные с тревожным дистрессом (Cohen's d = 0,16-0,39): «риск изоляции» и «возможное отсутствие лекарств для ежедневного приема». Особенно подверженными психологическому стрессу оказались страдающие аффективными расстройствами, лица молодого возраста ( $\leq 20$ лет), безработные, холостые/незамужние, не имеющие высшего образования и женщины. Таким образом, широкие слои населения нуждаются в коррекции дистрессовых опасений на фоне пандемии COVID-19, поэтому их проведение должно быть адресным, ориентированным по степени охвата и содержанию на выявленные уязвимые социальные группы.
\end{abstract}

Ключевые слова: коронавирусная инфекция, пандемия, COVID-19, психическое здоровье, тревога, аффективные расстройства, ассоциированная стигма

Благодарности: членам Российского общества психиатров, Анастасии Петровой и АНО «Партнерство равных», Марии Фаворской и ассоциации «Биполярники», Виктору Лебедеву и проекту «Дело Пинеля», а также просветительскому порталу «Психиатрия \& Нейронауки».

Вклад авторов: М. Ю. Сорокин - разработка дизайна исследования, статистическая обработка и анализ полученных данных, подготовка текста; Е. Д. Касьянов - разработка дизайна исследования, сбор материала, анализ полученных данных, подготовка текста; Г. В. Рукавишников - разработка дизайна исследования, анализ полученных данных, подготовка текста; О. В. Макаревич - разработка дизайна исследования, подготовка текста; Н. Г. Незнанов - редактирование; Н. Б. Лутова, Г. Э. Мазо - разработка концепции и дизайна исследования, анализ полученных данных, подготовка текста, редактирование.

Соблюдение этических стандартов: исследование проведено в соответствии с Хельсинкской декларацией Всемирной медицинской ассоциации (2013). Все участники дали согласие на обработку персональных данных перед включением в исследование.

$\triangle$ Для корреспонденции: Михаил Юрьевич Сорокин

ул. Бехтерева, д. 3, г. Санкт-Петербург, 192019; m.sorokin@list.ru

Статья получена: 06.05.2020 Статья принята к печати: 21.05.2020 Опубликована онлайн: 02.06.2020

DOI: 10.24075/vrgmu.2020.030

\section{STRUCTURE OF ANXIETY ASSOCIATED WITH COVID-19 PANDEMIC: THE ONLINE SURVEY RESULTS}

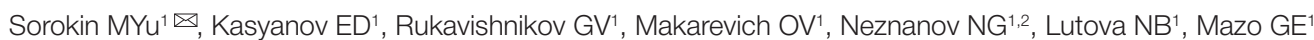

${ }^{1}$ V.M. Bekhterev National Medical Research Center for Psychiatry and Neurology, Saint-Petersburg, Russia

2 Pavlov First Saint Petersburg State Medical University, Saint-Petersburg, Russia

The COVID-19 pandemic imposed not only serious threats to the physical health of the population, but also provoked a wide range of psychological problems The study was aimed to define the structure of anxiety in the population during the epidemic period, as well as to identify the most vulnerable social groups (including individuals with affective disorders) which were most in need of psychological and/or psychiatric help. The online survey of 1957 Russian-speaking respondents aged over 18 was carried out from March 30 to April 5, 2020. The anxiety distress level was verified using the Psychological Stress Measure (PSM-25), the stigmatization of individuals experiencing respiratory symptoms was assessed using the modified Perceived Devaluation-Discrimination Questionnaire (PDD; Cronbach's $\alpha=0.707)$. In $99.8 \%$ of respondents, the combination of various concerns associated with COVID-19 was observed, the mean psychological stress score was increased to moderate level (score 104.9 \pm 34.4 ), and the stigmatization score exceeded the whole sample median value (19.5 \pm 3.4 ; Me $=17$ ). About $35 \%$ of respondents had concerns associated with anxiety distress (Cohen's $d=0.16-0.39$ ): these were the "risk of social isolation" and the "possible lack of medication for daily use". The following groups of respondents were the most susceptible to the stress: people with affective disorders, young people (aged $\leq 20$ ), unemployed persons, single persons, people with no formal education, and women. Thus, the broad sectors of the population need correction of anxiety distress associated with the COVID-19 pandemic. Therefore, the measures' implementation should be targeted, and in terms of coverage and content oriented to the identified vulnerable social groups.

Keywords: coronavirus infection, pandemic, COVID-19, mental health, anxiety, affective disorders, associated stigma

Acknowledgements: we would like to express our gratitude to the Russian Society of Psychiatrists (RSP) members, to Anastasia Petrova and the Partnership of Equal ANPO, to Maria Pushkina (Favorskaya) and the Bipolar association, to Victor Lebedev and the Pinel's Affair project, and to the Psychiatry \& Neurosciences educational service.

Author contribution: Sorokin MYu — research design, statistical processing and data analysis, manuscript writing; Kasyanov ED — research design, data acquisition and analysis, manuscript writing; Rukavishnikov GV — research design, data analysis, manuscript writing; Makarevich OV — research design, manuscript writing; Neznanov NG — editing; Lutova NB, Mazo GE — research concept and design, data analysis, manuscript writing and editing

Compliance with ethical standards: the study was performed in accordance with the World Medical Association Declaration of Helsinki (2013). All participants submitted the consent to personal data processing

Correspondence should be addressed: Mikhail Yu. Sorokin

Bekhtereva, 3, Saint-Petersburg, 192019; m.sorokin@list

Received: 06.05.2020 Accepted: 21.05.2020 Published online: 02.06.2020

DOI: 10.24075/brsmu.2020.030 
В ноябре 2019 г. впервые была зарегистрирована новая коронавирусная инфекция COVID-19 (от англ. coronavirus disease 2019), вызываемая коронавирусом SARS-CoV-2 [1]. Данная инфекция довольно быстро распространилась в Ухане (столице китайской провинции Хубэй) и затем по всему Китаю, перекинувшись на другие страны (в том числе Российскую Федерацию (РФ)), вызвав глобальную чрезвычайную ситуацию в области здравоохранения [2]. Уже 11 марта 2020 г. в связи с высокой распространенностью COVID-19 Всемирная организация здравоохранения (ВО3) признала текущую ситуацию пандемией [3]. В РФ первые лица с COVID-19 были выявлены 31 января 2020 г., а в начале апреля уже более 5000 россиян имели подтвержденные диагнозы [4, 5].

Пандемия COVID-19 создала серьезные угрозы для физического здоровья и жизни людей. Кроме того, опасность заражения коронавирусом вызвала среди населения стран с высоким уровнем распространения вирусной инфекции широкий спектр психологических проблем, таких как паническое расстройство, тревога и депрессия [6]. С марта 2020 г. правительства многих стран ввели карантинные меры, чтобы ограничить распространение инфекции и минимизировать нагрузку на медицинские службы. Лицам старше 65 лет, лицам с сопутствующими заболеваниями и беременным было предложено изолировать себя от прямого контакта с людьми в течение как минимум 12 недель, а лицам с подозрением на носительство коронавируса было поручено оставаться в своих домах и изолировать себя и всех, кто живет с ними, в течение как минимум 14 дней [7].

Таким образом возникли условия, когда одновременно присутствует ряд факторов, оказывающих влияние на психическое здоровье населения:

1) беспрецедентная потенциально угрожающая жизни ситуация с неопределенной продолжительностью;

2) широкомасштабные карантинные меры во всех крупных городах, которые, по сути, ограничивают жителей пребыванием в своих домах;

3) неопределенный инкубационный период вирусной инфекции и ее возможная бессимптомная передача;

4) сообщения о нехватке медицинских средств защиты;

5) неустойчивый информационный фон с переизбытком противоречивой информации;

6) неопределенность, связанная с влиянием коронавирусной инфекции COVID-19 на экономическую ситуацию в стране.

Пандемия коронавирусной инфекции COVID-19, по данным китайских исследователей, спровоцировала параллельно идущую эпидемию тревожных и депрессивных реакций $[8,9]$. При этом определенные группы населения могут быть более уязвимыми к психологическому стрессу, ассоциированному с данным заболеванием. В первую очередь лица с афффективными расстройствами - они значительно более подвержены эмоциональным откликам на пандемию COVID-19, проявляющимся в рецидивах уже имеющихся психических расстройств или ухудшении состояния, В силу высокой чувствительности к стрессу в сравнении с общей популяцией, а также в связи с ограничением планового амбулаторного приема врачейпсихиатров. Кроме того, помимо возрастания уровня переживаемого стресса среди населения возрастают стигма и дискриминация по отношению к определенным группам населения [10], даже при отсутствии доказательств об увеличении риска заболеваемости среди дискриминируемых групп.
Целью исследования было выявить структуру тревожных переживаний населения в период эпидемии и определить наиболее уязвимые социальные группы (в том числе среди лиц с аффективными расстройствами), больше всего нуждающиеся в психологической и/или психиатрической помощи.

\section{МАТЕРИАЛЫ И МЕТОДЫ}

\section{Получение данных}

Данные для исследования были получены на основании онлайн-опроса, проведенного с 30 марта по 5 апреля 2020 г. Участникам исследования предлагали заполнить анкету через интернет-платформу Google Forms, что в среднем требовало около 15 мин. Анкету распространяли в социальных сетях, а также на сайтах общественных организаций и тематических сообществ (см. Благодарности).

Критерии включения: способность читать на русском языке, наличие согласия на обработку персональных данных, фактом которого считали заполнение всех предложенных форм опроса. Критерии исключения (определены максимально свободно с целью репрезентации возможно большего числа социальных групп среди респондентов): 1) возраст участников < 18 лет; 2) наличие незаполненных разделов анкеты.

Анкета включала социодемограсические данные респондентов, а также сведения о наличии/ отсутствии аффективных расстройств (депрессивного расстройства, биполярного аффективного расстройства, генерализованного тревожного расстройства, дистимии и циклотимии), соматической патологии на основании самоотчетов участников исследования.

Респондентам предлагали отметить любое количество из предложенных 10 пунктов анкеты, описывающих различные варианты опасений в связи с пандемией COVID-19, а также любое число среди шести поведенческих вариантов предотвращения заражения, которые они практиковали (полная анкета представлена в Приложении). Отдельно респонденты могли определить, с какой частотой они обращались за информацией о пандемии в течение последней недели, если ее ранжировать на восемь градаций в диапазоне от «ни разу» до «ежечасно». Для оценки тревожного дистресса были использованы вопросы Шкалы психологического стресса (PSM-25) [11]. На основе широко распространенного опросника воспринимаемой стигматизации, его раздела обесценивания/дискриминации (PDD) [12] нами были сформулированы утверждения, описывающие предвзятое отношение к лицам, имеющим признаки простудного заболевания (кашель, насморк, чихание). Степень согласия с утверждениями этого опросника респонденты оценивали по четырехбалльной шкале Лайкерта, и большее суммарное значение соответствовало интенсивной стигме.

\section{Статистический анализ}

Статистическую обработку данных проводили с помощью программного продукта SPSS-16 (SPSS Inc., США). Использовали дескриптивные статистики. Проверку на нормальность распределения осуществляли по методу оценки асимметрии. Дисперсионный анализ для данных с номинальными шкалами проводили с использованием критерия $\chi^{2}$ Пирсона, с порядковыми шкалами - по U-критерию Манна-Уитни. Для групп, различающихся на 
уровне статистической значимости $p \leq 0,05$, вычисляли размеры эффектов по критериям Cohen's d и Cramer's V. При сопоставлении номинальных признаков с более чем двумя градациями интерпретацию размера эффекта проводили с поправкой на число степеней свободы и указанием пороговых значений для слабого/умеренного/ сильного эффекта. Высчитывали корреляционные коэффициенты Спирмена. Оценку внутренней согласованности оригинального опросника стигматизации проводили с использованием коэффициента $\alpha$ Кронбаха.

\section{РЕЗУЛЬТАТЫ ИССЛЕДОВАНИЯ}

В финальный реестр вошли 2117 записей, сделанных в течение первой недели рекомендуемого в России режима самоизоляции (с 30 марта по 5 апреля). Из анализа были исключены данные 160 респондентов, не соответствующих возрастному критерию. Таким образом, статистический анализ был проведен для данных опроса 1957 респондентов.

\section{Демографические показатели}

Большую часть выборки составили женщины - 1649 человек (84,3\%). Средний возраст респондентов достигал 31 года ( $\mathrm{Me}=27 ; \mathrm{Q}_{25}=22, \mathrm{Q}_{75}=38$ ). В выборку вошли респонденты из городов федерального значения - СанктПетербурга (21,1\%), Москвы (16,8\%) - и всех федеральных округов России (57,6\%), а также стран ближнего и дальнего зарубежья (4,5\%).

\section{Социальные показатели}

Около половины всех респондентов имели высшее образование (55,3\%). О наличии неоконченного высшего образования сообщили 25,6\% опрошенных. Значительная часть респондентов на момент опроса работала в частных $(23,6 \%)$ и государственных $(32,2 \%)$ организациях. До 10,3\% выборки составили медицинские работники. Не имели постоянного места работы 22,2\% респондентов. Холосты или не замужем были 51,8\% респондентов. В зарегистрированном браке состояли 26,9\% респондентов, в незарегистрированном - 12,4\%.

\section{Сопутствующие заболевания}

Наличие сопутствующей соматической патологии было указано в 54,8\% случаев. О наличии тех или иных аффективных расстройств сообщили 29,5\% выборки. Наиболее часто респонденты указывали депрессивное и биполярное аффективное расстройства (19,8\%), реже тревожные расстройства (6,0\%) и циклотимию или дистимию $(3,7 \%)$.

\section{Характеристика психологических и поведенческих реакций респондентов}

Проведенный корреляционный анализ всего массива данных показал, что попытки приспособления к новым условиям жизни в период распространения COVID-19 представляют собой многоуровневый процесс, имеющий сложную структуру взаимосвязанных факторов. Большее количество практикуемых мер предотвращения заражения короновирусом (в среднем - четыре: $\mathrm{Me}=4 ; \mathrm{Q}_{25}=3$, $\left.\mathrm{Q}_{75}=4\right)$ и частый поиск информации об эпидемии (в среднем двукратное в течение дня: $\mathrm{Me}=6 ; \mathrm{Q}_{25}=5, \mathrm{Q}_{75}=7$ ) предсказуемо коррелировали друг с другом и были связаны интенсификацией психологических реакций участников исследования в ответ на пандемию: увеличивались число сюжетов опасений о COVID-19, уровень связанного с ними психологического стресса, а также склонность респондентов стигматизировать лиц с респираторными симптомами. Практически все перечисленные показатели тоже были чувствительны к социально-демографическим параметрам обследованной выборки (табл. 1).

Среди участников исследования 99,8\% сообщали о наличии как минимум двух сюжетов для волнения по поводу коронавируса, наиболее распространенное число тем тревожных переживаний было равно пяти $\left(\mathrm{Me}=5 ; \mathrm{Q}_{25}=4\right.$, $\mathrm{Q}_{75}=6$ ) (табл. 2). Разнообразие тревожных реакций было ассоциировано с показателем психологического стресса (PSM-25), достигающим по всей выборке уровня средней интенсивности 104,9 балла (Me = 106; $\mathrm{Q}_{25}=80, \mathrm{Q}_{75}=$ 130). Качественный анализ взаимосвязи отдельных тем переживания о COVID-19 с уровнями психологического стресса и стигматизации-дискриминации лиц с респираторными симптомами показал разнонаправленные эффекты отдельных опасений.

Наличие опасения о жизни и здоровье близких не было ассоциировано со статистически значимым нарастанием уровня стресса или существенным увеличением стигмы, что, вероятно, определялось максимальной охваченностью этим переживанием подавляющего числа респондентов. В то же время с клинически значимым эффектом нарастания психологического стресса (слабым по магнитуде) было связано наличие двух наиболее редко встречавшихся из

Таблица 1. Взаимосвязь социально-демографических характеристик с психологическими и поведенческими реакциями респондентов в отношении COVID-19

\begin{tabular}{|c|c|c|c|c|c|}
\hline & $\begin{array}{c}\text { Частота поиска } \\
\text { информации о COVID-19 }\end{array}$ & $\begin{array}{c}\text { Число сюжетов } \\
\text { волнения о COVID-19 }\end{array}$ & $\begin{array}{c}\text { Число мер предотвращения } \\
\text { COVID-19 }\end{array}$ & Стресс (PSM-25) & Стигматизация \\
\hline Возраст & $0,06^{\star \star \star}$ & $-0,23^{\star \star \star}$ & - & $-0,38^{\star \star \star}$ & $0,06^{\star *}$ \\
\hline Образование & $0,08^{* * *}$ & $-0,15^{\star \star \star}$ & - & $-0,22^{\star \star \star}$ & - \\
\hline $\begin{array}{l}\text { Частота поиска информации } \\
\text { o COVID-19 }\end{array}$ & 1,000 & - & - & - & - \\
\hline $\begin{array}{l}\text { Число сюжетов волнения } \\
\text { o COVID-19 }\end{array}$ & $0,22^{\star \star \star}$ & 1,000 & - & - & - \\
\hline $\begin{array}{l}\text { Число мер предотвращения } \\
\text { COVID-19 }\end{array}$ & $0,17^{\star \star \star}$ & $0,30^{\star \star *}$ & 1,000 & - & - \\
\hline Стресс по PSM-25 & $0,14^{\star \star *}$ & $0,28^{\star * \star}$ & $0,05^{\star}$ & 1,000 & - \\
\hline Стигматизация & $0,10^{\star \star \star}$ & $0,12^{* * *}$ & $0,12^{\star \star \star}$ & - & 1,000 \\
\hline
\end{tabular}

Примечание: корреляционные коэффициенты Spearman's rho; $N=1957 ;{ }^{\star}-p \leq 0,05 ;{ }^{* \star}-p \leq 0,01 ;{ }^{* \star \star}-p \leq 0,001$. 
Таблица 2. Типология опасений о COVID-19 с сопутствующими уровнями тревожного дистресса и выраженности стимгатизации лиц с респираторными симптомами

\begin{tabular}{|c|c|c|c|}
\hline \multirow{2}{*}{ Тип присутствующего волнения } & \multirow{2}{*}{ Представленность (человек/доля в \%) } & \multicolumn{2}{|c|}{ Изменение уровней (SE): } \\
\hline & & стресса & стигмы \\
\hline Риск для жизни и здоровья родственников и близких & $1527 / 77,2$ & $-{ }^{*}$ & $+0,06$ \\
\hline Возможные материальные трудности & $1128 / 57,0$ & $+0,16$ & $-0,04$ \\
\hline Тяжелые социальные последствия & $980 / 49,5$ & $+0,14$ & $-0,08$ \\
\hline Отсутствие специального лечения COVID-19 & 789 / 39,9 & $+0,1$ & $+0,19$ \\
\hline Невозможность привычного образа жизни & $766 / 38,7$ & $+0,17$ & $-0,16$ \\
\hline Заразность вируса & $708 / 35,8$ & $+0,1$ & $+0,27$ \\
\hline Опасность для собственной жизни & $619 / 31,3$ & $+0,14$ & $+0,36$ \\
\hline Отсутствие в продаже средств защиты & $544 / 27,5$ & $+0,16$ & $+0,23$ \\
\hline Возможное отсутствие лекарств для ежедневного приема & $434 / 21,9$ & $+0,39$ & $+0,19$ \\
\hline Риск изоляции & $351 / 17,7$ & $+0,43$ & $-0,14$ \\
\hline
\end{tabular}

Примечание: размер эффекта (SE) слабый при 0,2 $\leq$ Cohen's $d \leq 0,49 ; p \leq 0,05$.

10 сюжетов для волнения: «возможного отсутствия лекарств для ежедневного приема" и "риска изоляции" (табл. 2). В совокупности среди участников исследования 688 человек (35\% выборки) сообщали о наличии хотя бь одного из них.

Средний суммарный показатель опросника стигматизации лиц с респираторными симптомами достигал 19,5 балла при $\mathrm{Me}=17\left(\mathrm{Q}_{25}=15, \mathrm{Q}_{75}=19\right)$ и достаточной внутренней согласованности инструмента (коэффициент $\alpha$ Кронбаха - 0,707). При этом «риск изоляции» был связан с достоверным снижением склонности респондентов к стигматизации лиц с респираторными симптомами. Однако эффекты нарастания стигмы достигали уровня практической значимости в случаях, когда у участников исследования присутствовало волнение на темы «заразности вируса», его "опасности для собственной жизни» или «отсутствия в продаже средств защиты».

\section{Психологические реакции отдельных групп населения}

Среди отдельных групп респондентов конкретные сюжеть тревожных переживаний имели свои особенности. Два типа наиболее ассоциированных с психологическим стрессом сюжетов были присущи, в частности, лицам, сообщившим о наличии у них аффективных нарушений (табл. 3). Причем «риск изоляции» в большей степени вызывал опасения среди тех, кто имел сочетанную с аффективной соматическую патологию. В то же время «возможное отсутствие лекарств, необходимых для ежедневного приема», чаще волновало респондентов, страдающих аффективными расстройствами без соматической коморбидности.

Важно отметить, что среди 688 участников исследования, имеющих не один, а сочетание обоих ассоциированных с психологическим стрессом типов тревожных переживаний, респонденты без психических нарушений встречались столь же часто, как страдающие афффективными расстройствами. Неожиданным подтверждением внешней валидности онлайн-анкеты стало выявленное превалирование специфичного для лиц с тревожными расстройствами опасения за собственную жизнь, отличающего их от респондентов, имеющих патологию настроения (табл. 4).

Кроме традиционных групп населения, считающихся уязвимыми в отношении тревожных реакций, - страдающих аффективными и соматическими заболеваниями, гораздо более широкие слои населения демонстрировали различные превалирующие типы опасений о COVID-19. Так, женщины чаще мужчин переживали об отсутствии в продаже индивидуальных средств защиты, а также опасались за собственную жизнь (табл. 4). Более склонны опасаться изоляции были холостые и незамужние участники исследования, безработные и трудоустроенные в государственных учреждениях (табл. 5).

Существенно реже сюжет тревожных опасений по поводу риска изоляции встречался среди респондентов, имеющих высшее образование или ученую степень, а также лиц старше 31 года. Категорию участников опроса старше 60 лет выделяла большая склонность опасаться материальных трудностей, возможных на фоне развития пандемии (табл. 6).

\section{ОБСУЖДЕНИЕ РЕЗУЛЬТАТОВ}

На основании проведения онлайн-опроса нами были получены данные, которые позволяют оценить структуру психологических переживаний, характерных для русскоязычных респондентов на первой неделе рекомендуемого режима самоизоляции в России. Проведенный анализ показал широкое распространение

таблица 3. Специфика тревожных переживаний в зависимости от группы здоровья респондентов

\begin{tabular}{|c|c|c|c|c|c|c|}
\hline \multirow{2}{*}{\multicolumn{2}{|c|}{ Типы волнения о COVID-19 }} & \multirow{3}{*}{$\begin{array}{c}\begin{array}{c}\text { Здоровые } \\
n=643\end{array} \\
11,0 \%\end{array}$} & \multicolumn{3}{|c|}{ Наличие заболевания } & \multirow{4}{*}{$\begin{array}{c}\text { Критерий статистической } \\
\text { значимости } \\
x^{2}=63,8 ; p=0,000 \\
\text { SE }=0,25\end{array}$} \\
\hline & & & аффективного & соматического & коморбидно & \\
\hline \multirow{2}{*}{ Риск изоляции } & + & & $21,5 \%$ & $17,9 \%$ & $31,0 \%$ & \\
\hline & - & $89,0 \%$ & $78,5 \%$ & $82,1 \%$ & $69,0 \%$ & \\
\hline \multirow{2}{*}{$\begin{array}{l}\text { Недоступность } \\
\text { лекарств }\end{array}$} & + & $22,2 \%$ & $34,3 \%$ & $14,4 \%$ & $30,4 \%$ & \multirow{2}{*}{$\begin{array}{c}x^{2}=59,6 ; p=0,000 \\
\mathrm{SE}=0,21\end{array}$} \\
\hline & - & $77,8 \%$ & $65,7 \%$ & $85,6 \%$ & $69,6 \%$ & \\
\hline
\end{tabular}

Примечание: размер эффекта (SE) средний при 0,17 $\leq$ Cramers's $V \leq 0,29$. 
Таблица 4. Специфика тревожных переживаний в зависимости от типа аффективного расстройства и пола

\begin{tabular}{|c|c|c|c|c|}
\hline \multirow{2}{*}{\multicolumn{2}{|c|}{ Типы волнения о COVID-19 }} & \multicolumn{2}{|c|}{ Представленность по группам } & \multirow{2}{*}{$\begin{array}{c}\text { Критерий статистической } \\
\text { значимости }\end{array}$} \\
\hline & & 1 & 2 & \\
\hline \multicolumn{5}{|c|}{ Гендерный фактор: 1 - мужчины, 2 - женщины } \\
\hline \multirow{2}{*}{ Дефицит средств защиты } & + & $20,1 \%$ & $29,2 \%$ & \multirow{2}{*}{$\begin{array}{c}x^{2}=10,7 ; p=0,001 \\
\mathrm{SE}=0,14\end{array}$} \\
\hline & - & $79,9 \%$ & $70,8 \%$ & \\
\hline \multirow{2}{*}{ Опасность для собственной жизни } & + & $23,1 \%$ & $33,2 \%$ & \multirow{2}{*}{$\begin{array}{c}x^{2}=12,4 ; p=0,000 \\
\mathrm{SE}=0,14\end{array}$} \\
\hline & - & $76,9 \%$ & $66,8 \%$ & \\
\hline \multicolumn{5}{|c|}{ Тип аффективных нарушений: 1 - нарушения настроения, 2 - тревожные расстройства } \\
\hline \multirow{2}{*}{ Опасность для собственной жизни } & + & $26,7 \%$ & $40,7 \%$ & \multirow{2}{*}{$\begin{array}{c}x^{2}=8,8 ; p=0,003 \\
\text { SE }=0,12\end{array}$} \\
\hline & - & $73,3 \%$ & $59,3 \%$ & \\
\hline
\end{tabular}

Примечание: размер эффекта (SE) малый при 0,1 $\leq$ Cramers's $V \leq 0,3$.

среди участников исследования различных причин для тревожных переживаний в связи с пандемией COVID-19, которые в совокупности обеспечивали повышенный уровень психологического стресса в обследованной выборке.

На фоне изменившихся условий жизни и деятельности, связанных с карантинными мероприятиями, у респондентов ожидаемо ссормировались разнообразные опасения, вызванные пандемией COVID-19. Важно отметить, что переживания по поводу «риска для жизни и здоровья родственников и близких» не приводили к нарастанию психологического стресса, в силу чего их можно рассматривать в рамках адаптационных личностных и психологических реакций. Одновременно расширение списка сюжетов волнений приводило к срыву адаптационных механизмов, провоцируя интенсификацию как психологического напряжения, проявляющегося в усилении тревоги, так и социального, когда переживаемый стресс сознательно или неосознанно перемещался во вне, вызывая усиление стигмы. Важно подчеркнуть, что психологический стресс особенно нарастал на фоне опасений в связи с «возможным отсутствием лекарств для ежедневного использования» и «риском изоляции», что в первом случае, по-видимому, связано с ухудшением субъективного восприятия, а во втором - с тем, что сами по себе карантинные мероприятия провоцируют волну тревоги и гнева. Нарастание стигматизационных установок в наибольшей степени оказалось связано со следующими переживаниями: «опасность для собственной жизни», «заразность вируса» и "отсутствие в продаже средств защиты», по-видимому, в большей степени обусловленных ощущением утраты контроля за ситуацией.

Отдельного внимания заслуживают данные, полученные от респондентов, сообщивших о наличии у них аффективных расстройств. Для них, как и для лиц, не сообщивших о каких-либо психических нарушениях, были характерны те же типы наиболее ассоциированных с психологическим стрессом сюжетов - «риск изоляции» и «недоступность лекарств». Однако «риск изоляции» в большей степени вызывал опасения среди тех, кто имел сочетанную с аффективной соматическую патологию. В то же время «возможное отсутствие лекарств, необходимых для ежедневного приема» чаще волновало респондентов, страдающих аффективными расстройствами без соматической коморбидности. Кроме того, для лиц с тревожными расстройствами было характерно превалирование специфичного для них «опасения за собственную жизнь», в сравнении с больными аффективными расстройствами, что подчеркивает клиническое своеобразие их переживаний.

Полученные данные о структуре тревожных переживаний респондентов позволяют выделить их особенности среди разных групп населения, что важно для формирования дифференцированных программ оказания психологической и социальной помощи. В частности, волнения по поводу «риска изоляции» в наибольшей степени свойственны молодым респондентам (моложе 31 года), относящимся к холостым или незамужним, не имеющим высшего образования и безработным, а также лицам с сочетанной аффективной и соматической патологией. Если в первых трех случаях это может быть обусловлено личностной незрелостью, несформированными навыками самоконтроля и самозанятости, а также временной утратой возможности общения, то для безработных основная причина - это снижение возможности для материального обеспечения. Для лиц старшей возрастной группы особой темой волнений оказались «материальные трудности»,

Таблица 5. Специфика тревожных переживаний в группах респондентов по их занятости и семейному статусу

\begin{tabular}{|c|c|c|c|c|c|c|c|}
\hline \multirow{2}{*}{\multicolumn{2}{|c|}{$\begin{array}{l}\text { Тип волнения } \\
\text { о COVID-19 }\end{array}$}} & \multicolumn{5}{|c|}{ Представленность по группам: } & \multirow{2}{*}{$\begin{array}{l}\text { Критерий статистической } \\
\text { значимости }\end{array}$} \\
\hline & & 1 & 2 & 3 & 4 & 5 & \\
\hline \multicolumn{8}{|c|}{$\begin{array}{c}\text { Трудовой статус: } 1 \text { - обучение, } n=271 ; 2-\text { безработные, } n=435 ; 3-\text { частный наем, } n=462 ; 4-\text { государственный наем, } n=631 ; \\
5-\text { собственный бизнес, } n=158\end{array}$} \\
\hline \multirow{2}{*}{ Риск изоляции } & + & $19,6 \%$ & $25,1 \%$ & $8,2 \%$ & $20,9 \%$ & $12,0 \%$ & \multirow{2}{*}{$\begin{aligned} x^{2}= & 52,6 ; p=0,000 \\
& \mathrm{SE}=0,19\end{aligned}$} \\
\hline & - & $80,4 \%$ & $74,9 \%$ & $92,8 \%$ & $79,1 \%$ & $88,0 \%$ & \\
\hline \multicolumn{8}{|c|}{$\begin{array}{c}\text { Семейный статус: } 1 \text { - вдовцы/вдовы, } n=30 ; 2-\text { разведенные, } n=144 ; 3-\text { холостые/незамужние, } n=1014 ; 4-\text { незарегистрированный брак, } \\
\qquad n=243 ; 5-\text { зарегистрированный брак, } n=526\end{array}$} \\
\hline \multirow{2}{*}{ Риск изоляции } & + & $3,3 \%$ & $13,2 \%$ & $22,5 \%$ & $16,9 \%$ & $11,8 \%$ & \multirow{2}{*}{$\begin{aligned} x^{2}= & 34,5 ; p=0,000 \\
& \mathrm{SE}=0,16\end{aligned}$} \\
\hline & - & $96,7 \%$ & $86,8 \%$ & $77,5 \%$ & $83,1 \%$ & $88,2 \%$ & \\
\hline
\end{tabular}

Примечание: размер эффекта (SE) средний при 0,15 $\leq$ Cramers's $V \leq 0,25$. 
ORIGINAL RESEARCH I PSYCHIATRY

Таблица 6. Специфика тревожных переживаний в зависимости от образования и возраста

\begin{tabular}{|c|c|c|c|c|c|c|c|c|}
\hline \multirow{2}{*}{\multicolumn{2}{|c|}{$\begin{array}{l}\text { Тип волнения о } \\
\text { COVID-19 }\end{array}$}} & \multicolumn{6}{|c|}{ Представленность по группам } & \multirow{2}{*}{$\begin{array}{l}\text { Критерий статистической } \\
\text { значимости }\end{array}$} \\
\hline & & 1 & 2 & 3 & 4 & 5 & 6 & \\
\hline \multicolumn{9}{|c|}{$\begin{array}{c}\text { Образование: } 1 \text { - неоконченное среднее, } n=31 ; 2-\text { среднее, } n=98 ; 3-\text { профессиональное, } n=164 ; 4 \text { - неоконченное высшее, } n=501 ; \\
5 \text { - высшее, } n=1082 ; 6 \text { - степень, } n=81\end{array}$} \\
\hline \multirow{2}{*}{ Риск изоляции } & + & $25,8 \%$ & $24,5 \%$ & $22,6 \%$ & $24,0 \%$ & $14,5 \%$ & $6,2 \%$ & \multirow{2}{*}{$\begin{aligned} x^{2}=35,1 ; p=0,000 \\
\mathrm{SE}=0,14\end{aligned}$} \\
\hline & - & $74,2 \%$ & $75,5 \%$ & $77,4 \%$ & $76,0 \%$ & $82,5 \%$ & $93,8 \%$ & \\
\hline \multicolumn{9}{|c|}{ Возраст: $1-18-20$ лет, $\mathrm{n}=310 ; 2-21-30$ лет, $n=859 ; 3-31-40$ лет, $n=363 ; 4-41-50$ лет $n=231 ; 5-51-60$ лет, $n=136 ; 6-60-78$ лет, $n=58$} \\
\hline \multirow{2}{*}{ Риск изоляции } & + & $28,7 \%$ & $20,0 \%$ & $14,3 \%$ & $8,2 \%$ & $8,8 \%$ & $12,1 \%$ & \multirow{2}{*}{$\begin{aligned} x^{2}= & 54,0 ; p=0,000 \\
& S E=0,18\end{aligned}$} \\
\hline & - & $71,3 \%$ & $80,0 \%$ & $85,7 \%$ & $91,8 \%$ & $91,2 \%$ & $87,9 \%$ & \\
\hline \multirow{2}{*}{$\begin{array}{l}\text { Материальные } \\
\text { трудности }\end{array}$} & + & $31,3 \%$ & $32,2 \%$ & $30,6 \%$ & $29,9 \%$ & $30,9 \%$ & $39,7 \%$ & \multirow{2}{*}{$\begin{array}{l}x^{2}=101,6 ; p=0,000 \\
\quad \mathrm{SE}=0,13\end{array}$} \\
\hline & - & $68,7 \%$ & $67,8 \%$ & $69,4 \%$ & $70,1 \%$ & $69,1 \%$ & $60,3 \%$ & \\
\hline
\end{tabular}

Примечание: размер эффекта (SE) средний при 0,13 $\leq$ Cramers's $V \leq 0,22$.

что очевидным образом диктует необходимость иных информационных и социальных интервенций.

В стратегическом плане ВОЗ по обеспечению готовности и реагирования на COVID-19 еще не определены какиелибо стратегии, направленные на возникшие потребности в области психического здоровья [13], хотя необходимость в них с большой долей вероятности будет возрастать как в процессе эпидемии, так и после ее завершения.

В доступной нам научной литературе отсутствуют данные о психологических реакциях на начальных этапах ухудшения эпидемиологической обстановки и объявления карантинных мероприятий как факта официального признания нарастания эпидемиологического неблагополучия. В Китае, который первым столкнулся с вопросами организации всех уровней медицинской помощи при распространении коронавируса, был разработан Принцип обеспечения поддержки психического благополучия. Он включал: 1) определение актуального статуса психического здоровья у населения; 2) определение круга лиц с высоким риском суицида и агрессии; 3) разработку структурированных мер помощи [14]. Но результативность оказания психологической помощи в этом регионе была признана недостаточной, что связывали с отсутствием опыта обучению принципам сохранения психического здоровья [15].

Таким образом, размеры и социальная гетерогенность группы риска обусловливают необходимость использовать для преодоления социально-психологических последствий пандемии широкие социальные интервенции, которые могут быть реализованы по принципу эшелонирования помощи: этап психо-социальной поддержки, специальной психологической помощи, а также клинико-психологическая помощь с привлечением психиатров. Как видно из опыта организации подобной работы в Китае, а также полученных нами данных, одним из препятствий организации эффективной поддержки населения может быть стигматизация/дискриминация [16].

Ограничения. Результаты исследования получены на основании самоотчетов респондентов. При том что степень корреляции самоотчетов с результатами объективных экспериментально-психологических обследований обычно бывает достаточно высока, дополнительная верификация анкетных сведений в ходе личной экспертной оценки исследователем могла бы повысить объективность полученных данных. В то же время подобная перекрестная оценка существенно ограничила бы размер выборки, представленность различных социальных групп, в том числе за счет переживания стигмы респондентами с аффективными расстройствами, кратно увеличила бы период получения первых результатов. Кроме того, в условиях настоятельной рекомендации социального дистанцирования возможность проведения очных визитов, которые предполагают посещение респондентом клиники, крайне ограничена. К тому же скорость развития эпидемического процесса и возникновения психологических реакций населения в ответ на пандемию COVID-19 определяет важность оперативности проведения исследований и формулирования на их основе рекомендаций психолого-психиатрической помощи.

Следует отдельно отметить, что выявленная сила корреляций психологических реакций населения с практикуемыми мерами предотвращения заражения и частотой поиска информации о пандемии соответствовала лишь уровню слабой или умеренной ассоциации признаков. Подобная ситуация достаточно характерна для исследований психологии поведения человека. С одной стороны, это очевидным образом иллюстрирует недопустимость интерпретации корреляционной связи как причинно-следственной даже в рамках проведенного исследования, когда наблюдаемые признаки семантически тесно связаны. С другой стороны, выявленная сила связей подчеркивает многофакторный характер регуляции поведения, где ни один из параметров нельзя считать детерминирующим.

\section{ВЫВОДЫ}

Вмешательство в психологический кризис должно быть рассмотрено как часть ответных мер общественного здравоохранения на вспышку COVID-19. Первым шагом должно стать широкое привлечение авторитетных профессионалов в области инфекционных заболеваний, эпидемиологии и психического здоровья в СМИ с целью информирования граждан о действенных мерах по предупреждению развития инфекции и способах предотвращения развития стресса в противовес дилетантским суждениям, наводняющим Интернет. Кроме того, наши данные позволяют рекомендовать проведение коррекции дистрессовых опасений широко, но и более адресно, с преимущественным охватом лиц молодого возраста, женщин, лиц, не имеющих высшего образования, не занятых в трудовой или учебной деятельности, холостых и незамужних, а не только пациентов с аффективными расстройствами. Наиболее острая необходимость коррекции опасений о пандемии COVID-19 выявлена в случае возникновения беспокойства по поводу возможного отсутствия лекарств для ежедневного приема и 
риска изоляции, поскольку эти типы переживаний связань с максимальным нарастанием тревожного дистресса в обследованной выборке. Необходимы создание доступа к индивидуальному психологическому консультированию широких масс в онлайн-режиме на государственном уровне и обеспечение психолого-психиатрической помощи нуждающимся при адекватном обеспечении эпидемиологической безопасности, поскольку психологическая стабильность способствует сохранению и физического здоровья населения.

\section{Литература}

1. Chan JF-W, Yuan S, Kok KH, To KKW, Chu H, Yang J, et al. A familial cluster of pneumonia associated with the 2019 novel coronavirus indicating person-to-person transmission: a study of a family cluster. The Lancet. 2020; 395 (10223): 514-23. Available from: https://doi.org/10.1016/S0140-6736(20)30154-9.

2. Statement on the second meeting of the International Health Regulations (2005) Emergency Committee regarding the outbreak of novel coronavirus (2019-nCoV). World Health Organization (WHO), 2020. Available from (assessed Feb 15, 2020): https:// www.who.int/news-room/detail/30-01-2020-statement-onthe-second-meeting-of-the-international-health-regulations(2005)-emergency-committee-regarding-the-outbreak-of-novelcoronavirus-(2019-ncov)

3. "WHO Director-General's opening remarks at the media briefing on COVID-19". World Health Organization (WHO) (Press release), 11 March 2020. Archived from the original on 11 March 2020 Available from: https://www.who.int/dg/speeches/detail/whodirector-general-s-opening-remarks-at-the-media-briefing-oncovid-19---11-march-202.

4. В России выявили двух первых больных коронавирусом. Доступно по ссылке: https://www.interfax.ru/russia/693554.

5. COVID-19 Dashboard by the Center for Systems Science and Engineering (CSSE) at Johns Hopkins University (JHU). Available from: https://gisanddata.maps.arcgis.com/apps/opsdashboard/ index.html\#/bda7594740fd40299423467b48e9ecf6.

6. Qiu J, Shen B, Zhao M, et al A nationwide survey of psychological distress among Chinese people in the COVID-19 epidemic: implications and policy recommendations. General Psychiatry. 2020; 33: e100213.

7. Brooks SK, Webster RK, Smith LE, Woodland L, Wessely S, Greenberg N, et al. The Psychological Impact of Quarantine and How To Reduce It: Rapid Review of the Evidence. The Lancet. 2020; 395 (10227): 912-20. Available from: https://doi.
org/10.1016/S0140-6736(20)30460-8

8. Yao H, Chen JH, Xu YF. Patients with mental health disorders in the COVID-19 epidemic. Lancet Psychiatry. 2020; 7 (4): e21. DOI: 10.1016/S2215-0366(20)30090-0/.

9. Lai J, Ma S, Wang Y, Cai Z, Hu J, Wei N, et al. Factors Associated With Mental Health Outcomes Among Health Care Workers Exposed to Coronavirus Disease 2019. JAMA Netw Open. 2020; 3 (3): e203976. DOI: 10.1001/jamanetworkopen.2020.3976.

10. Addressing Stigma. Centers for disease control and prevention. Available from: https://emergency.cdc.gov/cerc/cerccorner/ article_123016.asp.

11. Водопьянова Н. Е. Психодиагностика стресса. СПб.: Питер, 2009; 336 c.

12. Link B, Cullen F, Frank J, Wozniak J. The Social Rejection of Former Mental Patients: Understanding Why Labels Matter. American Journal of Sociology. 1987; 92 (6): 1461-500. Retrieved April 12, 2020. Available from: www.jstor.org/stable/2779844.

13. Novel coronavirus (2019-nCoV): strategic preparedness and response plan Feb 3, 2020. World Health Organization, 2019 [cited 2020 Feb 7]. Available from: https://www.who.int/docs/ default-source/coronaviruse/srp-04022020.pdf.

14. Li W, Yang Y, Liu ZH, Zhao Y-J, Zhang Q, Zhang L, et al. Progression of Mental Health Services during the COVID-19 Outbreak in China. Int J Biol Sci. 2020; 16 (10): 1732-8. DOI: 10.7150/ijbs.45120.

15. Chen Q, Liang M, Li Y, Cuo J, Fei D, Wang L, et al. Mental health care for medical staff in China during the COVID-19 outbreak. Lancet Psychiatry. 2020; 7 (4): e15-e16. DOI: 10.1016/S22150366(20)30078-X.

16. Kang L, Li Y, Hu S, Chen M, Yang C, Yang BX, et al. The mental health of medical workers in Wuhan, China dealing with the 2019 novel coronavirus. Lancet Psychiatry. 2020; 7 (3): e14. DOI: 10.1016/S2215-0366(20)30047-X

\section{References}

1. Chan JF-W, Yuan S, Kok KH, To KKW, Chu H, Yang J, et al. A familial cluster of pneumonia associated with the 2019 novel coronavirus indicating person-to-person transmission: a study of a family cluster. The Lancet. 2020; 395 (10223): 514-23. Available from: https://doi.org/10.1016/S0140-6736(20)30154-9.

2. Statement on the second meeting of the International Health Regulations (2005) Emergency Committee regarding the outbreak of novel coronavirus (2019-nCoV). World Health Organization (WHO), 2020. Available from (assessed Feb 15, 2020): https:// www.who.int/news-room/detail/30-01-2020-statement-onthe-second-meeting-of-the-international-health-regulations(2005)-emergency-committee-regarding-the-outbreak-of-novelcoronavirus-(2019-ncov)

3. "WHO Director-General's opening remarks at the media briefing on COVID-19". World Health Organization (WHO) (Press release), 11 March 2020. Archived from the original on 11 March 2020 Available from: https://www.who.int/dg/speeches/detail/whodirector-general-s-opening-remarks-at-the-media-briefing-oncovid-19---11-march-202.

4. V Rossii vyyavili dvuh pervyh bol'nyh koronavirusom. Available from: https://www.interfax.ru/russia/693554. Russian.

5. COVID-19 Dashboard by the Center for Systems Science and Engineering (CSSE) at Johns Hopkins University (JHU). Available from: https://gisanddata.maps.arcgis.com/apps/opsdashboard/ index.html\#/bda7594740fd40299423467b48e9ecf6.

6. Qiu J, Shen B, Zhao M, et al A nationwide survey of psychological distress among Chinese people in the COVID-19 epidemic: implications and policy recommendations. General Psychiatry. 2020; 33: e100213.

7. Brooks SK, Webster RK, Smith LE, Woodland L, Wessely S, Greenberg N, et al. The Psychological Impact of Quarantine and How To Reduce It: Rapid Review of the Evidence. The Lancet. 2020; 395 (10227): 912-20. Available from: https://doi. org/10.1016/S0140-6736(20)30460-8.

8. Yao H, Chen JH, Xu YF. Patients with mental health disorders in the COVID-19 epidemic. Lancet Psychiatry. 2020; 7 (4): e21. DOI: 10.1016/S2215-0366(20)30090-0/.

9. Lai J, Ma S, Wang Y, Cai Z, Hu J, Wei N, et al. Factors Associated With Mental Health Outcomes Among Health Care Workers Exposed to Coronavirus Disease 2019. JAMA Netw Open. 2020; 3 (3): e203976. DOI: 10.1001/jamanetworkopen.2020.3976.

10. Addressing Stigma. Centers for disease control and prevention. Available from: https://emergency.cdc.gov/cerc/cerccorner/ article_123016.asp.

11. Vodopjanova NE. Psihodiagnostika stressa. SPb.: Piter, 2009; 336 s. Russian.

12. Link B, Cullen F, Frank J, Wozniak J. The Social Rejection of Former Mental Patients: Understanding Why Labels Matter. 


\section{ORIGINAL RESEARCH I PSYCHIATRY}

American Journal of Sociology. 1987; 92 (6): 1461-500. Retrieved April 12, 2020. Available from: www.jstor.org/stable/2779844.

13. Novel coronavirus (2019-nCoV): strategic preparedness and response plan Feb 3, 2020. World Health Organization, 2019 [cited 2020 Feb 7]. Available from: https://www.who.int/docs/ default-source/coronaviruse/srp-04022020.pdf.

14. Li W, Yang Y, Liu ZH, Zhao Y-J, Zhang Q, Zhang L, et al. Progression of Mental Health Services during the COVID-19 Outbreak in China. Int J Biol Sci. 2020; 16 (10): 1732-8. DOI: 10.7150/ijbs.45120.

15. Chen Q, Liang M, Li Y, Cuo J, Fei D, Wang L, et al. Mental health care for medical staff in China during the COVID-19 outbreak. Lancet Psychiatry. 2020; 7 (4): e15-e16. DOI: 10.1016/S22150366(20)30078-X.

16. Kang L, Li Y, Hu S, Chen M, Yang C, Yang BX, et al. The mental health of medical workers in Wuhan, China dealing with the 2019 novel coronavirus. Lancet Psychiatry. 2020; 7 (3): e14. DOI: 10.1016/S2215-0366(20)30047-X. 